\title{
VOLUMETRIC CELLS: A FRAMEWORK FOR A BIO-INSPIRED GEOMETRIC MODELLING METHOD TO SUPPORT HETEROGENEOUS LATTICE STRUCTURES
}

\author{
N. Letov ${ }^{\otimes}$ and Y. F. Zhao \\ McGill University, Canada \\ $\bowtie$ nikita.letov@mail.mcgill.ca
}

\begin{abstract}
Current geometrical modelling approaches are unable to handle complex geometrical objects such as heterogeneous lattice structures. In this work, a framework for a novel bio-inspired geometric modelling method is proposed. The method can potentially support geometric modelling of heterogeneous lattice structures. The method utilises discretisation algorithms that are based on cell division processes encountered in nature. The method is verified on two 2D use-cases.
\end{abstract}

Keywords: 3D modelling, additive manufacturing, biologically inspired design, lattice structures

\section{Introduction}

Humanity has always been striving to extend the design freedom to achieve a larger design potential. The Additive Manufacturing (AM) technique can serve as a disruptive fabrication process that provides an enormous design freedom. AM is defined as "the process of joining materials to make objects from 3D model data, usually layer upon layer, as opposed to subtractive manufacturing methodologies" (ASTM, 2015). It is believed that AM technology is bringing the fourth industrial revolution which would introduce a new era of connected enterprises (Hermann et al., 2016). Indeed, AM does not require any extra tooling and can build a part with complexity unachievable by conventional manufacturing methods (Chu et al., 2008). Nowadays, AM is mostly applied for parts consolidations, weight reduction, functional customization, personalization and aesthetics (Wohlers and Gornet, 2014) and prompts research in numerous fields: computational optimization, geometric modelling, behavioural simulation, material science, etc. (ISO, 2015). The so-called lattice structures have complex geometrical properties that are hard or even impossible to be produced without AM (Vayre et al., 2012). In this research, a lattice structure is defined as "an architecture formed by an array of spatial periodic unit cells with edges and faces" (Tao and Leu, 2016). Lattice structures provide not only an optimal performance-to-weight ratio (Frulloni et al., 2007) but also unique physical properties, e.g. increased energy-absorption, vibrational damping, etc. (Wadley, 2002).

Lattice structures can be classified as homogeneous and heterogeneous (see Figure 1). In heterogeneous lattice, the thickness of struts or nodes inside of it varies over the entire structure, while these parameters stay constant in homogeneous lattice (Tang et al., 2019). Thus, geometric modelling of heterogeneous lattice, rather than homogeneous, represents a bigger challenge. 

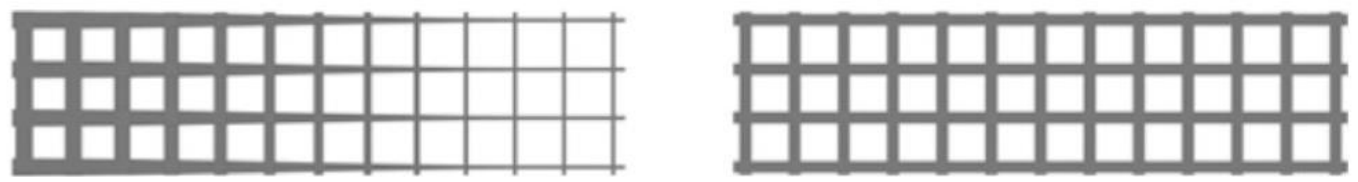

Figure 1. A heterogeneous (left) and homogeneous (right) lattice structures (Tang et al., 2019)

The evolution process made the simplest organisms on Earth converge to complicated and robust species such as humans. It resulted in the optimal shapes and structures that are parts of living organisms developed in billions of years. A scutoid introduced by Gómez-Gálvez et al. (2018) is the example of a unit cell that forms a biological structure by specific tessellations. This begs a question: can unit cells used in geometric modelling be of any shape, e.g., a prism, a scutoid, etc.? Moreover, as the idea of lattice structures comes directly from nature, the approach proposed in this work aims to guarantee that the geometric modelling method itself should be bio-inspired. Indeed, some lattice structures are not only non-periodic, but also conformal (Tang et al., 2016). In nature, scutoids form a cylindrical shape which is also close to conformal.

The content structure is organised as follows. In Section 2, concepts and existing challenges related to this work are reviewed. In Section 3, the proposed approach is presented and then verified in Section 4. In Section 5, the summary of this work is made, and future prospects are identified. In Section 6, the acknowledgments are presented.

\section{Overview of related concepts}

Geometric modelling of lattice structures has certain challenges which are mostly related to geometrical complexity of lattice and inability of conventional Computer-Aided Design (CAD) approaches to handle this complexity accordingly (Tang and Zhao, 2016). In this work, these challenges and concepts related to the work are reviewed.

\subsection{Multi-scale geometric modelling of heterogeneous lattice structures}

The ability to perform multi-scale modeling is crucial in many research fields involving computer graphics and geometric modeling. For example, Prada et al. (2017) applied multi-scale modeling concepts for 3D mesh generation process for multiscale bone analysis. Engquist et al. (2005) identified the need for an appropriate multi-scale geometric modeling tool for porous materials.

In heterogeneous lattice structures, optimization of structural topology and thickness of lattice struts results in meeting the given functional requirements (Tang et al., 2015). Heterogeneity of a structure provides unique properties that cannot be provided by homogeneous structures, e.g. gradual elasticity of the structure or structural stiffness (Martínez et al., 2016). Heterogeneous lattice structures usually have multi-scale complexity which cannot be handled by conventional geometric modelling tools. For heterogeneous lattice structures, it is required to consider not only the whole structure in its meso-scale, but also each joint and strut of the lattice as they form at micro-scale (Zhang et al., 2010). Geometric modelling in engineering is mainly used for visualizing engineering systems such as parts, assemblies, etc., and represented by CAD software. While conventional CAD software is normally sufficient for engineering design in industry, there is no geometric modelling tool that would be able to appropriately represent complex heterogeneous lattice structures due to inability of existing geometric modelling tools to represent a model in multi-scale, which forms a research gap that is yet to be filled. Another issue is the computational cost of such geometric modelling tools because heterogeneous lattice structures have high geometrical complexity resulting in high computational needs using conventional methods (Dong et al., 2017).

Multi-scale geometric modeling methods are also critical in medical research. For example, Youssef (2013) introduced a bio-inspired framework for the simulation of 3-D multicellular tissue growth. However, there is no geometric modeling tool to implement this framework due to high geometric complexity. The need for such a geometric modeling approach for bio-related research is identified, e.g. in anatomy even small defects within a model are crucial (Sacks et al., 2017). 


\subsection{Level of detail}

In computer graphics, manipulation of the level of detail (LOD) allows to avoid rendering of unnecessary details of an object on screen (Marschner and Shirley, 2016). However, decreasing LOD of a lattice structure down to a certain level can result in complete homogenization of lattice, such as removing all geometrical features corresponding to lattice structures from view (PTC, 2019).

Normally, LOD is manually or automatically associated with CAD-features of solid bodies, e.g. extrusions, revolutions, etc. (Borrmann et al., 2015). However, lattice structures are designed not with CAD-features but with nodes connected by struts which are not so well-defined from the CAD-perspective (Vayre et al., 2012). Moreover, in heterogeneous lattice even periodicity of the lattice is not well-defined.

\subsection{Volumetric modelling}

One more topic worth considering when discussing multi-scale geometric modelling is the application of multi-scale volumetric modeling. Volumetric modelling allows direct transition to structural simulations. Currently, there are three major approaches for volumetric modelling: voxel modelling, volumetric mesh, and functional representation (FRep). This work does not cover the FRep method as it is incompatible with other modelling formats and cannot store topology information which makes it troublesome to apply in AM (Pasko et al., 2011).

\subsubsection{Voxel modelling}

Voxelised models have an advantage in terms of downsampling and acquisition of real-world data, i.e. any geometrical complexity is feasible which, however, comes at a cost due to rise of computational resources required for rendering and inability to handle zooming efficiently (Laine and Karras, 2011). Since lattice structures are hard or even impossible to be produced without additive manufacturing techniques, there is no need in voxel size higher than 3D-printer resolution (Telea and Jalba, 2011).

In general, a voxel has a cubic shape for the sake of render simplicity. Cubic shape of a voxel also fits well for simulations. For example, a standard cubic unit element is usually considered for the theoretical framework of stress analysis within a structure. However, representing curved surfaces using cubic voxels introduces a distortion which gets removed as the number of cubes approaches infinity, which is not feasible from the computational point of view (Kaufman et al., 1993). This distortion affects simulations. For example, a cylinder segment is considered for the theoretical framework of stress analysis within a cylindrical solid (Xu et al., 2013).

There has been an attempt represent complex geometry with voxels other than of cubic shape, such as body-centred cubic (BCC) and face-centred cubic (FCC) (Strand, 2004). However, a voxelised model obtained with such voxels appears sparser due to a larger distance between the centres of two neighbouring voxels. Note that no research had been conveyed on combining different shapes of voxels, similarly to cells of different forms emerging into a single biological structure. Such research would contribute to not only additive manufacturing of heterogeneous lattice structures but also to 4D printing technique, i.e. additive manufacturing with smart materials (Sossou et al., 2019).

One of the most popular voxel-based simplification methods involves using sparse voxel octrees which are based on generating multi-scale voxels which could be visible or invisible depending on the resolution and size of the screen (Laine and Karras, 2011). Recent researches show that octree-based neural networks can be applied for 3D shape analysis and learning and thus can be adapted for feature recognition (Zeng et al., 2019). There is evidence that sparse voxel octrees can be used in AM (Zeng et al., 2019).

\subsubsection{Volumetric mesh}

Finite volume modelling (FVM) is similar to finite element modelling (FEM) used in the majority of CAD-software with the difference in discretizing the whole solid body and not only its surface, i.e. the whole body is considered to be subdivided with polyhedrons instead of polygon (Rom and Brakhage, 2011). For instance, FVM applied to such complex shapes as macromolecules provides sufficient visual information (Feng et al., 2013). FVM, similarly to FEM and unlike voxel-based methods, provides the freedom of unit cell orientation. This freedom enables the application of FVM in geometric modelling of 
conformal and Voronoi-based lattice structures (Tang et al., 2019). However, the computation of curvature values of polygon and polyhedron meshes is non-trivial due to their discrete nature. Moreover, a finite volume is always convex since it is bounded by a finite number of planes. Therefore, FVM introduces significant distortion when applied to non-convex geometric shapes such as lattice structures and surfaces with genus 1 and higher (Tewari et al., 2006). This implies the need in a proper meshing algorithm that takes convexity and curvature into the account and affects quality of meshed models, especially ones requiring multi-scale modelling (Seemann et al., 2016).

Summarising the above overview, there is a gap between geometrical complexity of heterogeneous lattices and inability of current volumetric geometric modelling methods to handle such complexity. The potential of using bio-inspired algorithms is pointed out in Section 1 and leads to the proposed approach.

\section{The proposed approach}

This research aims to develop a new geometric modelling approach that would support lattice structures, especially heterogeneous lattices. There are certain similarities between existing volumetric models and living organisms: they all consist of tiny 'blocks' that form their structure. However, there is a difference in the variety of shapes of those 'blocks'. For example, while voxels in a single model have the same shape (usually cubic), living organisms consist of cells with a whole variety of shapes (ellipsoids, polyhedrons, scutoids, etc.). Currently, the sparse voxel octree technique allows modelling with voxels of different sizes (Laine and Karras, 2011). However, the cubic shape of voxels remains the same which does not allow the variety of shapes that is present in nature and introduces anisotropy which depends on the orientation of cubic grid (Laine and Karras, 2011).

Similar to heterogeneous lattice structures, bio-inspired cellular materials lack an appropriate CAD software for their modelling as they require mesh- or voxel-based multi-scale geometric modelling approach (Savio et al., 2018). Note that cell geometry is not necessarily cubic, but also cylindrical, bacilli-like, etc. (Savio et al., 2018). For bio-inspired geometric modelling, other bio-inspired geometry classes must be considered.

\subsection{The proposed geometric model definition}

Before diving into the approach, in this work a general framework for quantifying volumetric geometric model is presented. Let $C$ be a set that corresponds to the geometric model of a (heterogeneous) structure that could be mapped to a set of $n$ unit elements that form it, i.e. $C(p) \mapsto$ $\left\{V_{1}, \ldots, V_{n}\right\}=\bigcup_{i=1}^{n} V_{i}$ where $p$ is a vector of lattice properties (for example, topology type, material, etc.), $\left\{V_{i}\right\}$ for $i=1, \ldots, n$ is a set of $n$ unit elements that correspond to the geometric model $C$, which are denoted in this work as volumetric cells (see Figure 2). First of all, $C$ is essentially a 3D-shape that is bounded by an orientable 2-manifold of finite genus, not necessarily convex. Secondly, each volumetric cell is defined, according to the complex cell theory, as a compact 3-manifold of genus 0 . Therefore, $C$ can be defined as a 3D quotient space which is the result of the quotient map that attaches volumetric cells $\left\{V_{i}\right\}$ by their boundaries $\partial V_{i}^{j}$ such that $\partial V_{i}^{j}$ is the $j$-th boundary of the $i$-th volumetric cells (Equation 1).

$$
C:=\bigcup_{i=1}^{n}\left(V_{i} / \bigcup_{j=1}^{m} \partial V_{i}^{j}\right) \subset \mathbb{R}^{3},
$$

This framework is generally the same for any other geometrical modelling method that involves discretising of volumetric data with, however, redefined constraints. First of all, unlike voxels, in the proposed approach, every volumetric cell $V_{i}$ could be different from each other both in shape and size. Secondly, unlike volumetric mesh, it is proposed that volumetric cells $V_{i}$ are not necessarily convex.

\subsection{The proposed geometrical modelling algorithms}

As the model discretisation is proposed to be bio-inspired in this work, it is important to investigate mechanisms that define geometrical shapes encountered in nature. In this work, two bio-inspired algorithms are developed to represent the most common biological geometric phenomena and then combined in a single algorithm. 


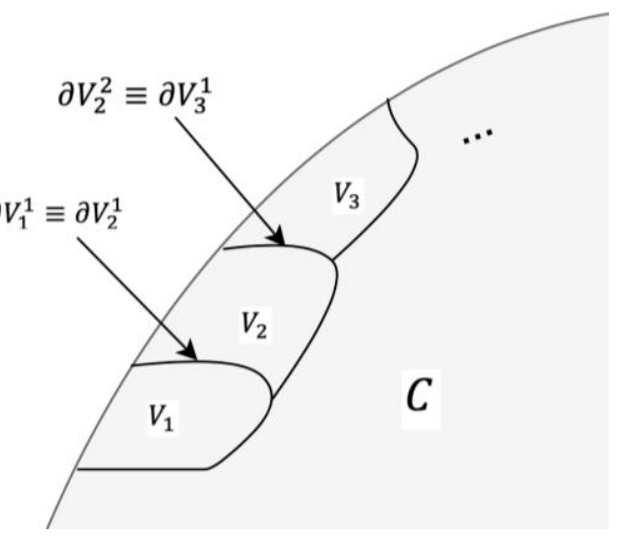

Figure 2. Representation of the geometrical model $C$

\subsubsection{The long axis rule}

One of most common patterns observed in cell geometry formation is the long axis rule (LAR) (see Figure 3). LAR defines the cleavage plane as the plane perpendicular to the longest axis passing though the center of mass (Minc et al., 2011).

In this work a geometric modelling approach that takes LAR as a base for its discretisation technique is proposed described as follows:

1. Let $\partial C$ be the boundary of a (heterogeneous) structure $C$. Set $k=1$. Set $k_{\max }$ to be the maximum number of iterations. Let $V_{1}^{k}$ be a single volume cell that fills the space bounded by $\partial C$.

2. Divide each volumetric cell $V_{l}^{k}$ into two volumetric cells $V_{2 l-1}^{k+1}$ and $V_{2 l}^{k+1}$ by LAR as follows:

a. For each $V_{l}^{k}$ identify its centroid $c=\frac{\int x F(x) d x}{\int F(x) d x}$ for all $x=\left(x_{1}, x_{2}, x_{3}\right) \in \mathbb{R}^{3}$ that are bounded by the function $F(x)$.

b. Identify the long axis $L$.

c. Identify the plane $P$ perpendicular to the long axis $L$.

d. Divide $V_{l}^{k}$ into two volumetric cells $V_{2 l-1}^{k+1}$ and $V_{2 l}^{k+1}$ by the plane $P$ such that $V_{2 l-1}^{k+1} \cap$ $V_{2 l}^{k+1}=P$ and $V_{2 l-1}^{k+1} \cup V_{2 l}^{k+1}=V_{l}^{k}$.

3. Set $k \leftarrow k+1$. If $k<k_{\max }$ go to Step 2 .

4. STOP.

Note that instead of centre of mass of a volumetric cell its centroid is used which is due to the fact that material of the structure is omitted, and only geometric properties are taken into the account. Note that LAR is not always followed in nature (see the bottom row in Figure 3).

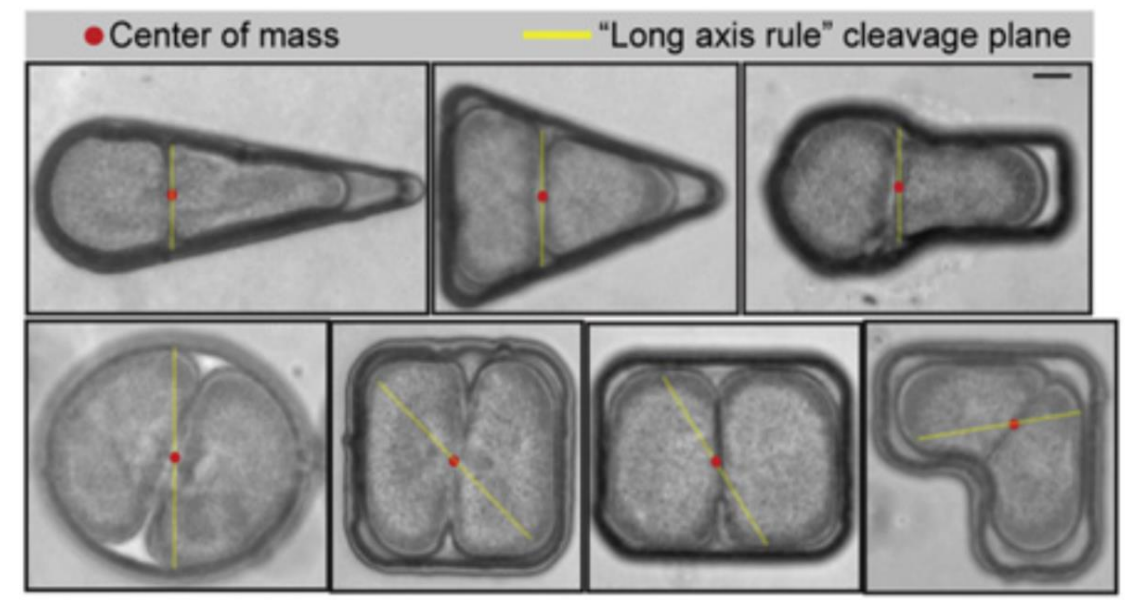

Figure 3. The long axis rule observed in cell division process in nature (Minc et al., 2011) 


\subsubsection{Surface-to-volume ratio}

It has been observed in nature that cells tend to minimize their surface-to-volume ratio ( $\mathrm{S}: \mathrm{V}$ ratio) (Schmick and Bastiaens, 2014). The 3D shape with the most optimal S:R ratio in this sense is a sphere which also explains the blob-like shape of most cells. Sometimes, external geometrical constraints (as well as the physical and environmental ones which, however, are omitted in this work due to irrelevance), do not allow sphericity (Marshall, 2011).

In this work a geometric modelling approach that takes the $\mathrm{S}: \mathrm{V}$ ratio as a base for its discretisation technique is proposed and described as follows:

1. Define $\partial C$ to be the boundary of a (heterogeneous) structure $C$. Set $k=1$. Set $k_{\max }$ to be the maximum number of iterations. Let $V_{1}^{k}$ be a single volume cell that fills the space bounded by $\partial C$.

2. Define the volumetric cells $V_{2 l-1}^{k+1}$ and $V_{2 l}^{k+1}$ by minimizing the $\mathrm{S}: \mathrm{V}$ ratio as follows:

a. For $V_{2 l-1}^{k+1}$ and $V_{2 l}^{k+1}$ define their $\mathrm{S}: \mathrm{V}$ functions defined as $R_{1}(x)=S_{1}(x) / V_{1}(x)$ and $R_{2}(x)=S_{2}(x) / V_{2}(x)$, respectively, where $S_{1,2}(x)$ is the surface and $V_{1,2}(x)$ is the volume of $V_{2 l-1}^{k}$ and $V_{2 l}^{k}$, respectively, both depending on cell division.

b. Find the optimal division surface $D(\hat{x})$ where $\hat{x}=\arg \min _{x}\left(R_{1}(x)+R_{2}(x)\right)$ and set $V_{2 l-1}^{k+1}$ and $V_{2 l}^{k+1}$ such that $V_{2 l-1}^{k+1} \cap V_{2 l}^{k+1}=D(\hat{x})$ and $V_{2 l-1}^{k+1} \cup V_{2 l}^{k+1}=V_{l}^{k}$. Note that $D(\hat{x})$ is not necessarily plane.

3. Set $k \leftarrow k+1$. If $k<k_{\max }$ go to Step 2 .

4. STOP.

Note that minimisation of $R_{1}(x)$ and $R_{2}(x)$ separately results in contradiction as $\min _{x} R_{1}(x)$ and $\min _{x} R_{2}(x)$ produce values of $\hat{x}$ such that either $V_{2 l-1}^{k+1}=V_{l}^{k}$ or $V_{2 l}^{k+1}=V_{l}^{k}$ while minimisation of $\left(R_{1}(x)+R_{2}(x)\right)$ is performed in a balanced way with no contradiction of values of $\hat{x}$.

\subsubsection{The combined approach}

At this stage of this work, the trade-off between two algorithms is performed manually, i.e. for each iteration of $k$ the division method is considered as given (see Figure 4). Note that the LOD concept can be applied to the result of the combined approach by combining neighbouring vertices of two adjacent volumetric cells and thus providing a modified version of the approach.

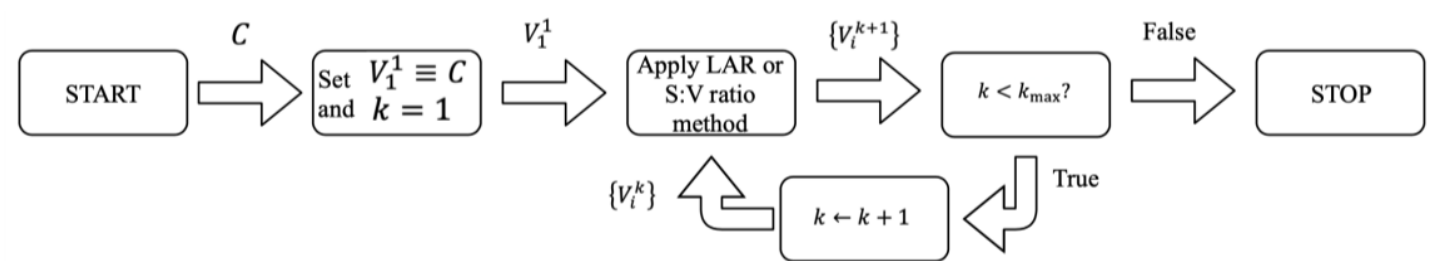

Figure 4. The flowchart of the proposed combined algorithm

\section{Verification}

In this work two use-cases are considered. For verification of the LAR method, a circular cross-section and a square cross-section with a hole in the middle are discretised into volumetric cells (see Figure 5a and Figure 6a, respectively). The hole in the middle is added as it is important to consider a simple shape with a non-zero genus as lattice structures have numerous holes. But every resulting cell is convex at every iteration of the algorithm a volumetric cell is divided into two by a plane. Moreover, in LAR there is high level of inconsistency between each volumetric cell, i.e. the variety of shapes is large.

The verification of the $\mathrm{S}: \mathrm{V}$ ratio method is performed similarly (see Figure $5 \mathrm{~b}$ and Figure 6b). Consider the first iteration $(k=1)$ applied to the circular cross-section with the set of $\mathrm{S}: \mathrm{V}$ functions defined (Equation 2).

$$
R_{1}(r)=2 / r \text { and } R_{2}(r)=\frac{2 R+2 r}{R^{2}-r^{2}} \text { for } r \in[0, R]
$$



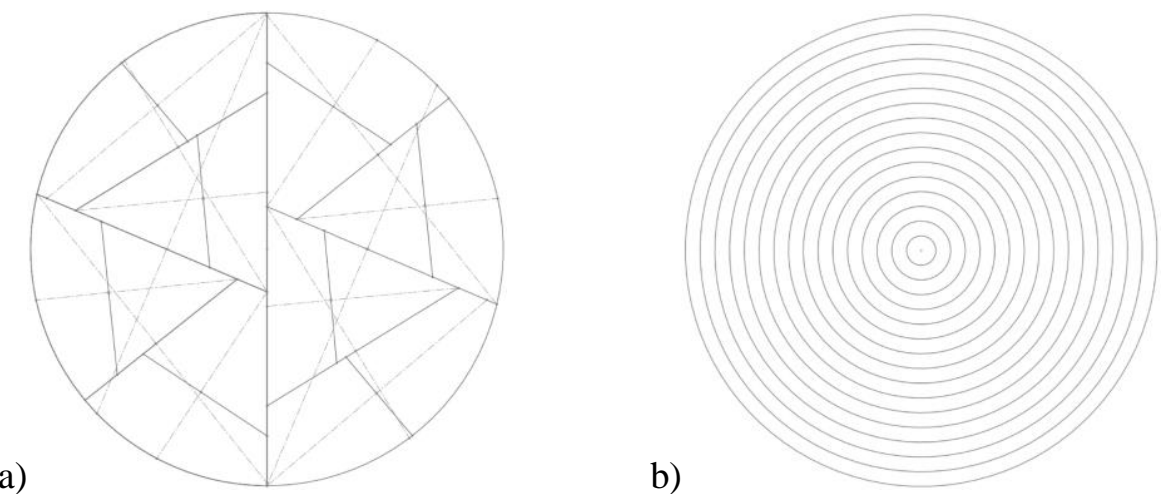

c)
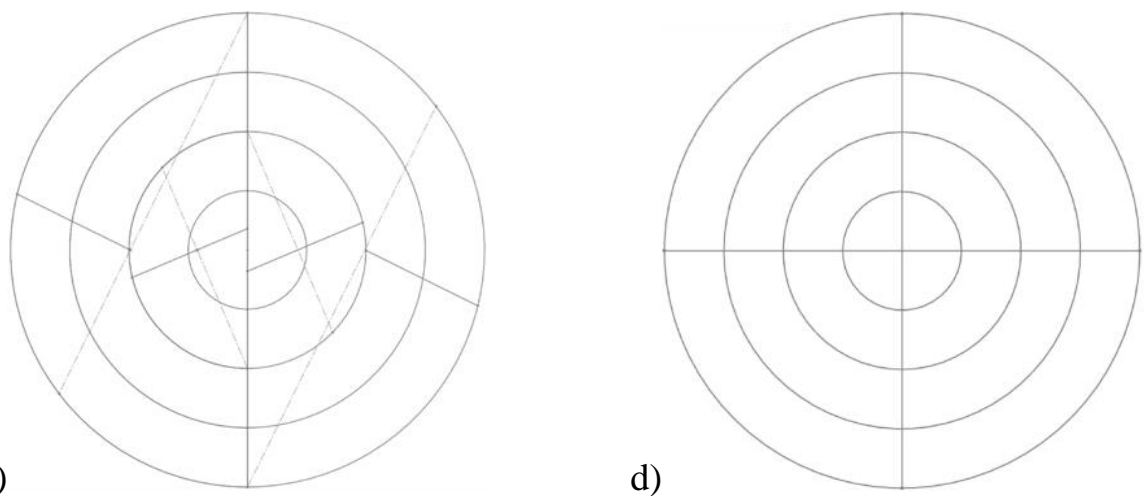

Figure 5. a) LAR, b) the S:V ratio minimisation, c) the combined method (left) and d) the modified combined algorithms applied as discretisation methods to a circular cross-section for $\boldsymbol{k}_{\max }=\mathbf{4}$ iterations. Long axes are shown as dotted lines

a)

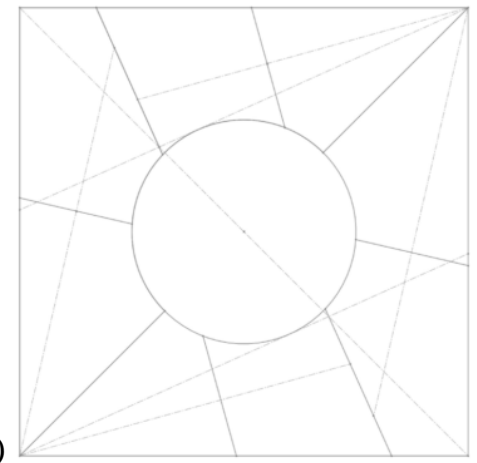

c)

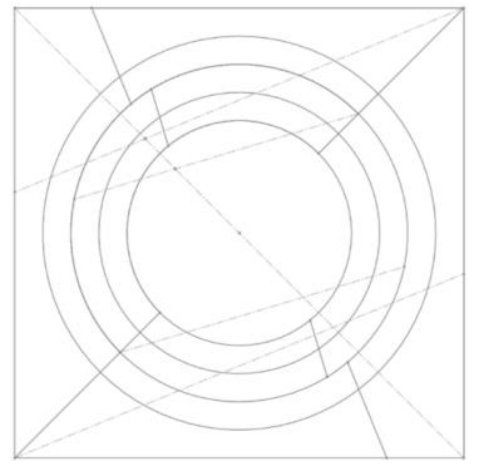

b)

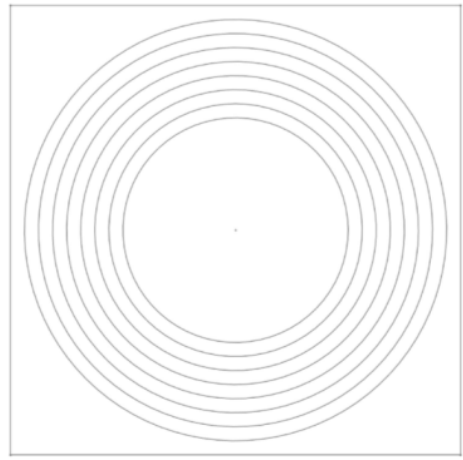

d)

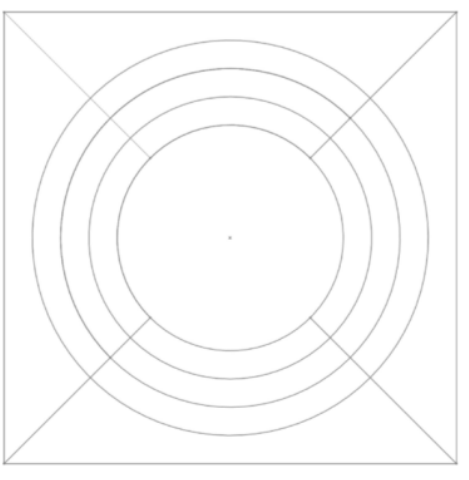

Figure 6. a) LAR, b) the S:V ratio minimisation, c) the combined method (left) and d) the modified combined algorithms applied as discretisation methods to a square cross-section with a circular hole in its middle for $\boldsymbol{k}_{\max }=\mathbf{4}$ iterations. Long axes are shown as dotted lines 
Note that $\arg \min _{r} R_{1}(x)=R$ and $\arg \min _{r} R_{2}(x)=0$, i.e. two values of $r$ contradict, while $\hat{r}=\arg \min _{x}\left(R_{1}(x)+R_{2}(x)\right)=R / 2$ produces the first optimal division surface $D(\hat{r})$. However, the resulting volumetric cells do not fit the framework as they correspond to 2-manifolds of genus higher than 0 .

In this work both LAR and S:V ratio methods are combined manually and verified for $k_{\text {max }}=4$ with $k=\{1,3\}$ corresponding to the LAR method and $k=\{2,4\}$ corresponding to the $\mathrm{S}: \mathrm{V}$ ratio method (see Figure $5 \mathrm{c}$ and Figure $6 \mathrm{c}$ ). Comparing to volumetric mesh, volumetric cells obtained with this method are not all convex; and comparing to voxelised models, volumetric cells discretise the model with similar unit cells but of different sizes. However, inconsistency between volumetric cells remains from the LAR method. This method is modified according to the LOD concept by combining neighbouring vertices of several pairs of adjacent volumetric cells (see Figure 5d and Figure 6d). This method provides the desired result which is similar to the unit cells used for stress analysis of cylindrical structures.

\section{Discussion and future work}

In this work a novel bio-inspired volumetric discretisation approach is proposed that has potential to support heterogeneous lattice structures. Geometric modelling kernels normally represent a curvature as a polygonal chain for rendering and computational efficiency. At this stage of the work, curvature representation is not considered, although must be taken into the account for future work. Note that the proposed method applied for the square cross-section acts as if the outer boundary is not present which is due to taking derivative of a circular inner boundary summed with the constant outer boundary. This effect needs to be considered in more detail for future research. Note that the $\mathrm{S}: \mathrm{V}$ ration method provides discretisation results that are similar to the results obtained by iterative application of the signed distance function (SDF) (Katopodes, 2018). Comparison of SDF with the S:V ratio method in combination with the LAR method shall be considered for future work.

Further development of this approach will be focused on:

1. Developing of a trade-off method between the LAR and the $\mathrm{S}: \mathrm{V}$ ratio algorithms, as the question of preference of some algorithm is still an open question in cellular biology (Minc et al., 2011).

2. As only one use-case has been considered in this work, it is proposed to consider more complicated use-cases. Moreover, as the ultimate goal of this novel approach is to support geometric modelling of heterogeneous lattice structures, it is crucial to implement this approach for 3D use-cases, which, eventually, shall include heterogeneous lattices themselves.

3. A comparison with other existing volumetric geometric modelling methods is required for $3 \mathrm{D}$ use-cases.

4. Investigation of other algorithms encountered in nature that define cellular geometry, e.g. fractal-based geometry is observed not only in trees and lungs, but also in Voronoi tessellations of cells (Mazurek, 2016).

5. General optimisation of the proposed approach as in long-term it should be computationally efficient enough to run on regular machines.

6. Implementing the LOD concept which is related to the number of iterations $k_{\max }$.

\section{Acknowledgements}

This research work is supported by National Sciences and Engineering Research Council of Canada Discovery Grant RGPIN-2018-05971.

\section{References}

ASTM (2015), ASTM F2792-12a: Standard terminology for additive manufacturing technologies, ASTM International, West Conshohocken (PA), United States.

Borrmann, A. et al. (2015), "Multi-scale geometric-semantic modeling of shield tunnels for GIS and BIM applications", Computer-Aided Civil and Infrastructure Engineering, Vol. 30 No. 4, pp. 263-281. https://doi.org/10.1111/mice.12090 
Chu, C., Graf, G. and Rosen, D.W. (2008), "Design for additive manufacturing of cellular structures", ComputerAided Design and Applications, Vol. 5 No. 5, pp. 686-696. https://doi.org/10.3722/cadaps.2008.686-696

Dong, G., Tang, Y. and Zhao, Y.F. (2017), “A Survey of Modeling of Lattice Structures Fabricated by Additive Manufacturing”, Journal of Mechanical Design, Vol. 139 No. 10, pp. 100906:1-100906:13. https://doi.org/ $10.1115 / 1.4037305$

Engquist, B., Lötstedt, P. and Runborg, O. (2005), Multiscale Methods in Science and Engineering, Springer, Berlin.

Feng, X. et al. (2013), "Multiscale geometric modeling of macromolecules II: Lagrangian representation", Journal of Computational Chemistry, Vol. 34 No. 24, pp. 2100-2120. https://doi.org/10.1002/jcc.23364

Frulloni, E. et al. (2007), "Experimental study and finite element analysis of the elastic instability of composite lattice structures for aeronautic applications", Composite Structures, Vol. 78 No. 4, pp. 519-528. https://doi.org/10.1016/j.compstruct.2005.11.013

Gómez-Gálvez, P. et al. (2018), "Scutoids are a geometrical solution to three-dimensional packing of epithelia", Nature Communications, Vol. 9 No. 1, pp. 2960. https://doi.org/10.1038/s41467-018-05376-1

Hermann, M., Pentek, T. and Otto, B. (2016), “Design principles for Industrie 4.0 scenarios", 2016 49th Hawaii International Conference on System Sciences (HICSS), IEEE, Kauai (HI), United States, pp. 3928-3937. https://doi.org/10.1109/HICSS.2016.488

ISO (2015), ISO/ASTM 17296-2:2015(E): Additive manufacturing — General Principles - Overview of process categories and feedstock, ISO, Geneva, Switzerland.

Katopodes, N.D. (2018), Free-Surface Flow, Elsevier. https://doi.org/10.1016/C2017-0-00542-6, available at.

Kaufman, A., Cohen, D. and Yagel, R. (1993), "Volume graphics", IEEE Computer, Vol. 26 No. 7, pp. 51-64. https://doi.org/10.1109/MC.1993.274942

Laine, S. and Karras, T. (2011), "Efficient sparse voxel octrees", IEEE Transactions on Visualization and Computer Graphics, Vol. 17 No. 8, pp. 1048-1059. https://doi.org/10.1109/TVCG.2010.240

Marschner, S. and Shirley, P. (2016), Fundamentals of Computer Graphics, Fourth Edition, CRC Press, New York (NY), United States.

Marshall, W.F. (2011), “Origins of cellular geometry”, BMC Biology, Vol. 9 No. 1, pp. 57. https://doi.org/ 10.1186/1741-7007-9-57

Martínez, J., Dumas, J. and Lefebvre, S. (2016), "Procedural voronoi foams for additive manufacturing", ACM Transactions on Graphics, Vol. 35 No. 4, pp. 44:1-44:12. https://doi.org/10.1145/2897824.2925922

Mazurek, P. (2016), "Cell structures modeling using fractal generator and torus geometry", 2016 21st International Conference on Methods and Models in Automation and Robotics (MMAR), IEEE, Miedzyzdroje, Poland, pp. 750-755. https://doi.org/10.1109/MMAR.2016.7575230

Minc, N., Burgess, D. and Chang, F. (2011), "Influence of cell geometry on division-plane positioning”, Cell, Vol. 144 No. 3, pp. 414-426. https://doi.org/10.1016/j.cell.2011.01.016

Pasko, A. et al. (2011), "Procedural function-based modelling of volumetric microstructures", Graphical Models, Vol. 73 No. 5, pp. 165-181. https://doi.org/10.1016/j.gmod.2011.03.001

Prada, D., Galvis, A. and Sollero, P. (2017), "Superficial 3D mesh generation process using multimedia software for multiscale bone analysis", International Conference on Boundary Element and Meshless Techniques, Bucharest, Romania, p. 126.

PTC. (2019), "Material homogenization for lattice simulation in additive manufacturing", available at: https://support.ptc.com/help/creo/creo_pma/r6.0/usascii/whats_new_pma/addmanumaterial_homogenization_lattice_simulation.html (accessed 22.10.2019).

Rom, M. and Brakhage, K.-H. (2011), Volume mesh generation for numerical flow simulations using Catmull-Clark and surface approximation methods, Institut für Geometrie und Praktische Mathematik, Aachen, Germany.

Sacks, M.S. et al. (2017), "On the need for multi-scale geometric modelling of the mitral heart valve", Healthcare Technology Letters, Vol. 4 No. 5, pp. 150. https://doi.org/10.1049/htl.2017.0076

Savio, G. et al. (2018), "Geometric modeling of cellular materials for additive manufacturing in biomedical field: A review”, Applied Bionics and Biomechanics, Vol. 2018, pp. 1654782:1-1654782:14. https://doi.org/ $10.1155 / 2018 / 1654782$

Schmick, M. and Bastiaens, P.I.H. (2014), "The interdependence of membrane shape and cellular signal processing”, Cell, Vol. 156 No. 6, pp. 1132-1138. https://doi.org/10.1016/j.cell.2014.02.007

Seemann, P. et al. (2016), "Simplification of multi-scale geometry using adaptive curvature fields", available at: http://arxiv.org/abs/1610.07368

Sossou, G. et al. (2019), "Design for 4D printing: A voxel-based modeling and simulation of smart materials", Materials and Design, Vol. 175, pp. 107798. https://doi.org/10.1016/j.matdes.2019.107798

Strand, R. (2004), "Surface skeletons in grids with non-cubic voxels", Proceedings of the 17th International Conference on Pattern Recognition, 2004. ICPR 2004., IEEE, Cambridge, UK, Vol. 1, pp. 548-551. https://doi.org/10.1109/ICPR.2004.1334195 
Tang, Y., Dong, G. and Zhao, Y.F. (2019), "A hybrid geometric modeling method for lattice structures fabricated by additive manufacturing", The International Journal of Advanced Manufacturing Technology, Vol. 102 No. 9-12, pp. 4011-4030. https://doi.org/10.1007/s00170-019-03308-x

Tang, Y., Kurtz, A. and Zhao, Y.F. (2015), "Bidirectional Evolutionary Structural Optimization (BESO) based design method for lattice structure to be fabricated by additive manufacturing", Computer-Aided Design, Vol. 69, pp. 91-101. https://doi.org/10.1016/j.cad.2015.06.001

Tang, Y., Yang, S. and Zhao, Y.F. (2016), "Design method for conformal lattice-skin structure fabricated by AM technologies", Volume 1A: 36th Computers and Information in Engineering Conference, American Society of Mechanical Engineers, Charlotte (NC), United States, p. V01AT02A037. https://doi.org/10.1115/ DETC2016-59738

Tang, Y. and Zhao, Y.F. (2016), "A survey of the design methods for additive manufacturing to improve functional performance", Rapid Prototyping Journal, Vol. 22 No. 3, pp. 569-590. https://doi.org/10.1108/ RPJ-01-2015-0011

Tao, W. and Leu, M.C. (2016), "Design of lattice structure for additive manufacturing", 2016 International Symposium on Flexible Automation (ISFA), IEEE, Cleveland (OH), United States, pp. 325-332. https://doi.org/10.1109/ISFA.2016.7790182

Telea, A. and Jalba, A. (2011), "Voxel-based assessment of printability of 3D shapes", Proceedings of the 10th International Conference on Mathematical Morphology and Its Applications to Image and Signal Processing, Springer-Verlag, Berlin, Germany, pp. 393-404. https://doi.org/10.1007/978-3-642-21569-8_34

Tewari, G., Gotsman, C. and Gortler, S.J. (2006), "Meshing genus-1 point clouds using discrete one-forms", Computers \& Graphics, Vol. 30 No. 6, pp. 917-926. https://doi.org/10.1016/j.cag.2006.08.019

Vayre, B., Vignat, F. and Villeneuve, F. (2012), "Designing for Additive Manufacturing", Procedia CIRP, Elsevier, Athens, Greece, Vol. 3, pp. 632-637. https://doi.org/10.1016/j.procir.2012.07.108

Wadley, H.N.G. (2002), “Cellular metals manufacturing”, Advanced Engineering Materials, Vol. 4 No. 10, pp. 726-733. https://doi.org/10.1002/1527-2648(20021014)4:10<726::AID-ADEM726>3.0.CO;2-Y

Wohlers, T.T. and Gornet, T. (2014), "History of additive manufacturing", Wohlers Report, Vol. 24 No. 2014 , pp. 118.

$\mathrm{Xu}$, B. et al. (2013), "A three-dimensional soil-water coupled FE analysis of hollow cylinder test concerning non-uniform deformation", Soils and Foundations, Vol. 53 No. 6, pp. 923-936. https://doi.org/10. 1016/j.sandf.2013.10.011

Youssef, B.B. (2013), "Parallelization of a bio-inspired computational model for the simulation of 3-D multicellular tissue growth", Procedia Computer Science, Elsevier, Baltimore (MD), USA, Vol. 20, pp. 391398. https://doi.org/10.1016/j.procs.2013.09.292

Zeng, J., Del Angel, A. and White, S. (2019), Generating data for a three-dimensional (3D) printable object, United States.

Zhang, H.W., Wu, J.K. and Fu, Z.D. (2010), "Extended multiscale finite element method for mechanical analysis of periodic lattice truss materials", International Journal for Multiscale Computational Engineering, Vol. 8 No. 6, pp. 597-613. https://doi.org/10.1615/IntJMultCompEng.v8.i6.40 